\section{OPEN ACCESS}

Edited by:

Gabriel Sandblom,

Karolinska Institutet (KI), Sweden

Reviewed by:

Hao Nguyen,

Bach Mai Hospital, Vietnam

Balasankar Ganesan,

The Chinese University of Hong Kong, China

${ }^{*}$ Correspondence: Le Nguyen Vu

nguyenvu.urologue@gmail.com

Tran Minh Tuan

bstuantran1978@gmail.com

Hoang-Long Vo

vohoanglonghmu@gmail.com

Specialty section:

This article was submitted to Visceral Surgery,

a section of the journal

Frontiers in Surgery

Received: 27 December 2020

Accepted: 15 July 2021

Published: 17 August 2021

Citation:

Vu LN, Nghia NQ, Tuan TM, Phuong TH, Vo H-L, Viet KN and

Giang TB (2021) Measuring

Health-Related Quality of Life in

Vietnamese Patients After Kidney

Transplantation

Front. Surg. 8:646629.

doi: 10.3389/fsurg.2021.646629

\title{
Measuring Health-Related Quality of Life in Vietnamese Patients After Kidney Transplantation
}

\author{
Le Nguyen $\mathrm{Vu}^{1 *}$, Nguyen Quang Nghia ${ }^{1}$, Tran Minh Tuan ${ }^{1 *}$, Tran Ha Phuong ${ }^{1}$, \\ Hoang-Long Vo ${ }^{2,3 *}$, Khai Ninh Viet ${ }^{1}$ and Tran Binh Giang ${ }^{1}$
}

' Organ Transplantation Center, Viet Duc University Hospital, Hanoi, Vietnam, ${ }^{2}$ Institute for Preventive Medicine and Public Health, Hanoi Medical University, Hanoi, Vietnam, ${ }^{3}$ Department of Scientific Research and International Cooperation, Hanoi Medical University Hospital, Hanoi, Vietnam

Objectives: To consider that the health-related quality of life (HRQOL) has become an inherent part of the patient outcomes in the care and treatment after kidney transplantation (KT). This study aimed to measure HRQOL among a representative sample size of patients after KT by using both the Short Form 36 (SF-36) and the Kidney Disease Quality of Life 36 (KDQOL-36).

Methods and Results: Data of this cross-sectional design were collected in the Organ Transplant Center, Viet Duc University Hospital (Hanoi, Vietnam) from January 2020 to March 2020 and included the patients aged 18 years or over after KT at 6 months, 1 year, and 3 years postoperatively. HRQOL was evaluated through face-to-face interviews by means of the SF-36 and KDQOL-36 measurement tools. According to the SF-36, the overall mean score of HRQOL was $69.13 \pm 15.55$ and the two domains were the highest scores of "Mental Health" $(81.23 \pm 14.28)$ and "General Health" $(80.06 \pm 14.81)$. When measuring with the KDQOL-36, the overall mean score was $68.67 \pm 13.75$ and was the highest in the domain "Symptoms and Problems of Kidney Disease" (87.06 \pm 16.00$)$. Both instruments had good reliability for those after KT. The reliability of the SF-36 was high with Cronbach's coefficients $\alpha=0.90$. There were positive relationships between the dimensions measured by the KDQOL-36 and SF-36 (correlation coefficient: 0.03-0.69). Similarly, the domains of the SF-36 also had positive correlations with the KDQOL-36 (correlation coefficient: 0.18-0.51). The correlation coefficient between overall HRQOL scores of the SF-36 and KDQOL-36 was 0.62 , indicating a strong correlation between the SF-36 and KDQOL-36.

Conclusions: There were slight fluctuations in the HRQOL score in domains in the 3-year follow-up stages, suggesting not having clear change. The mean SF-36 score was consistent with the mean KDQOL-36 score. High reliability and strong correlation were found between two instruments of the SF-36 and KDQOL-36. This study provides the reliability and constructs validity in the combination of two sets of the SF-36 and KDQOL-36 scales for the assessment of HRQOL among post-KT patients, thereby assisting physicians and health professionals in the clinical decision-making, assessment of therapeutic efficacy, and understanding of treatment risk.

Keywords: chronic kidney disease, kidney transplantation, quality of life, measurement, Vietnam 


\section{INTRODUCTION}

Health-related quality of life (HRQOL), a well-established aspect of health and general wellbeing, can be measured with a variety of instruments. In medicine, disease-specific HRQOL measures are designed to well reflect the HRQOL aspects of a particular disease (1). For chronic illness, physicians have often paid attention to HRQOL to measure the disease effects in their patients to better understand the impact level of an illness on the day-today life of a person. Chronic kidney disease (CKD), an important public health issue with the global increase, describes the gradual loss of kidney function in patients (2). CKD, in general, and end-stage kidney disease (ESKD), in particular, often lead to both physical and emotional symptoms that adversely impact the health and daily routine of patients (3). A large systematic study recently indicated that people with CKD have decreased quality of life and poorer socioeconomic circumstances as CKD progresses (2). Fortunately, the development and perfection of kidney replacement therapy (in the form of dialysis or kidney transplantation) have significantly improved survival rates in patients with ESKD, and HRQOL improves dramatically after the successful renal transplantation compared to patients maintained on the dialysis treatment (4). As the clinical practices have varied widely across the world with the increasing demands in many aspects, there needs to be an increased interest in the assessment of mental health aspects in patients with ESKD after kidney transplantation (KT).

In the context of the culture and value systems in each institution, routine use of HRQOL assessment may be useful in monitoring clinical transplant practice, informing treatment decision-making, and/or aiding in the allocation of transplant healthcare resources. Although the HRQOL advantages of renal transplantation are well established (5-7), large differences in quality of life are often observed depending on specific transplant cohorts. Importantly, most changes in the data in HRQOL have been reported from developed countries (3). Vietnam, a lower middle-income country, experiences rapid economic growth over the last decade with increasing social and healthcare outcomes (8). The current status posed a growing demand for end-stage renal failure management and KT in Vietnam (8). KT is still an emerging and challenging area in Vietnam, and insights on the aspects of HRQOL relevant to KT are needed to propose a suitable management strategy for post-KT patients. This study represents our largest effort at one institution in Vietnam to measure HRQOL among a representative sample size of patients after KT.

\section{METHODS}

\section{Study Design, Settings, and Participants}

This was a cross-sectional study carried out in the Organ Transplant Center, Viet Duc University Hospital (Hanoi, Vietnam) from January 2020 to March 2020. We included the patients who were monitored after KT at our institution at 6 months, 1 year, and 3 years postoperatively. We excluded the patients (i) suffered from kidney transplant failure after 3-month transplantation and must return to dialysis; (ii) died after KT; and (iii) experienced KT at our institution but post-KT followup treatment at other institutions. A total of 153 patients were eligible for the final analysis.

\section{Data Collection}

Written informed consent was obtained from participants prior to data collection. Data were collected by face-to-face interviews. Interviewers were the trained nurses at our institution. During the interview, HRQOL was assessed in studying patients according to the Short Form 36 (SF-36) and the Kidney Disease Quality of Life 36 (KDQOL-36).

\section{Health-Related Quality of Life SF-36}

The SF-36 is a generic health questionnaire measuring eight health domains, such as Physical Functioning (PF), Limitations in Daily Role Functioning due to Physical Problems (RP), Bodily Pain (BP), General Health (GH), Vitality (VT), Social Functioning (SF), Limitations in Daily Role Functioning due to Emotional Problems (RE), Mental Health (MH), and an item asking respondents about health change over the last year. Scores for each domain can range from 0 to 100 , higher scores indicating a better health state. Scores on each scale were calculated based on the "half item rule." Two summary scores were also calculated from the eight domains: the Physical Component Summary (PCS) and the Mental Health Component Summary (MCS) $(9,10)$.

\section{Kidney Disease Quality of Life 36}

The KDQOL-36 contains five subscales: Physical Component Summary (PCS), Mental Component Summary (MCS), Burden of Kidney Disease (BKD), Symptoms and Problems of Kidney Disease (SPKD), and Effects of Kidney Disease (EKD).

The KDQOL-36 has 36 items including the SF-12 version 1 (12 items total) and three kidney-specific scales (24 items total). The SF-12 yields the PCS and MCS, both of which are scored on a Tscore metric. The three kidney-targeted scales assess BKD, SPKD, and EKD. The Burden scale has four items (e.g., "My kidney disease interferes too much with my life") that are prompted with the context "How true or false is each of the following statements?" and have five response options that range from "Definitely true" to "Definitely false." The Symptoms/Problems scale has 12 items, each representing a symptom or side effect of kidney disease (e.g., "Washed out or drained?") that are given the context "During the past 4 weeks, to what extent were you bothered by each of the following?" and have five response options ranging from "Not at all bothered" to "Extremely bothered." The Effects scale has eight items (e.g., "Your ability to work around the house?") with the context "How much does kidney disease bother you in each of the following areas?" and the same response options as the Symptoms/Problems subscale. Each of these scales is scored by transforming all items to a 0-100 possible range and averaging across the items on each scale to create scale scores. The KDQOL-36 items are all scaled so that higher scores indicate better HRQOL. The previously published norms (unadjusted means) for these scales are Burden $=41$, Symptoms $/$ Problems $=71$, and Effects $=63$. Although 
TABLE 1 | Patient characteristics.

\begin{tabular}{lc}
\hline Characteristics $(\boldsymbol{n}=\mathbf{1 4 9})$ & Number (\%) \\
\hline Age (years) & \\
Mean (SD) & $39.48 \pm 10.76$ \\
IQR & $19-70$ \\
Gender & \\
Male & $114(74.51)$ \\
Female & $39(25.49)$ \\
Living area & \\
Rural & $57(37.25)$ \\
Urban & $96(62.75)$ \\
Comorbidity & \\
Diabetes & $11(7.19)$ \\
Hypertension & $34(23.78)$ \\
Others & $108(69.03)$ \\
Occupation & \\
Officer & $70(45.75)$ \\
Farmer & $17(11.11)$ \\
Freelancer & $45(29.41)$ \\
Student & $11(7.19)$ \\
Retirer & $10(6.54)$ \\
Education & \\
Lower secondary or lower & $10(6.54)$ \\
Upper secondary & \\
University or higher & $44(28.76)$ \\
Marital status & $99(64.71)$ \\
Single & \\
Married & $39(25.49)$ \\
Widowed/divorced & $107(69.93)$ \\
Mime on dialysis (months) & $7(4.58)$ \\
IQR & $38.45(88.33)$ \\
\hline &
\end{tabular}

the KDQOL-36 has been translated into more than 25 different languages, a valid Vietnamese translation version was published by RAND Health Care (11).

\section{Data Analysis}

Data were sorted, cleaned, coded, and entered into Epidata 3.1 and analyzed using Statistical Package for Stata 13.1 software. Socioeconomic characteristics were summarized using descriptive statistics. Quantitative data were expressed as mean, $\mathrm{SD}$, and interquartile range (IQR), whereas categorical data were expressed as frequency and percentage. Then, we calculated mean, SD, and Cronbach's alpha values of eight domains (PF, $\mathrm{RP}, \mathrm{BP}, \mathrm{GH}, \mathrm{VT}, \mathrm{SF}, \mathrm{RE}$, and $\mathrm{MH}$ ) for the SF-36 and of five domains (PCS, MCS, BKD, SPKD, and EKD) for the KDQOL36. The degree of association in overall HRQOL between the SF-36 and KDQOL-36 was measured by Pearson's correlation coefficient and denoted by r. Internal consistency reliability was evaluated using Cronbach's alpha coefficient calculated separately for each subscale. Coefficient alpha of 0.70 or greater is generally considered to be acceptable (12).

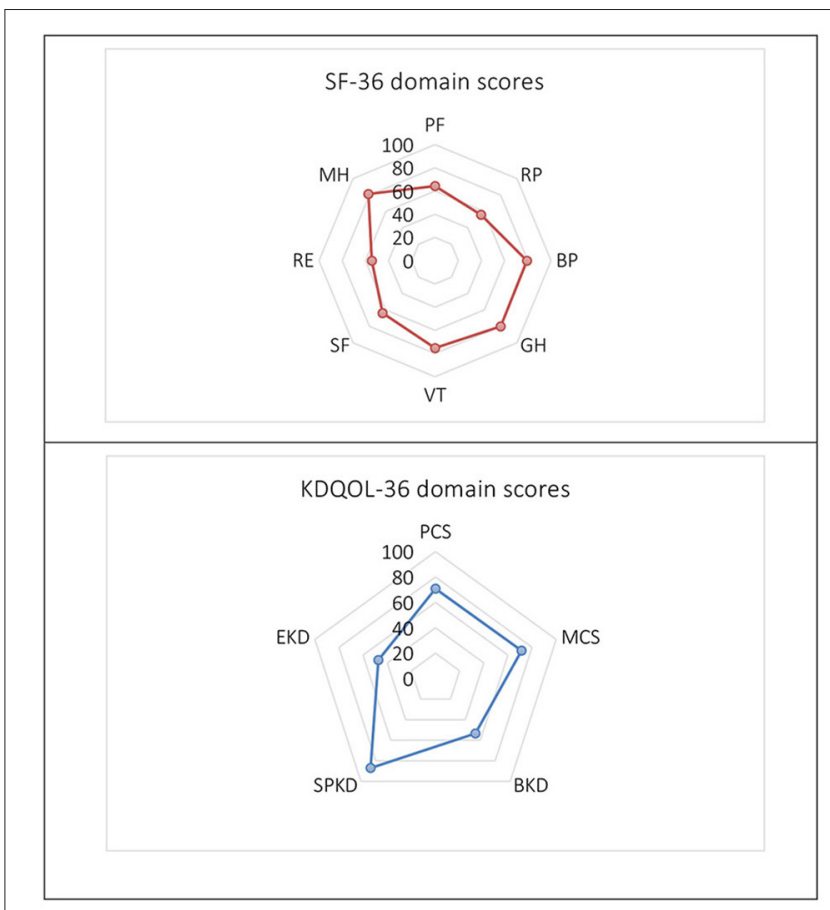

FIGURE 1 | The Short Form 36 (SF-36) and Kidney Disease Quality of Life 36 (KDQOL-36) domain scores. PF, Physical Functioning; RP, Limitations in Daily Role Functioning due to Physical Problems; BP, Bodily Pain; GH, General Health; VT, Vitality; SF, Social Functioning; RE, Limitations in Daily Role Functioning due to Emotional Problems; MH, Mental Health; PCS, Physical Component Summary; MCS, Mental Component Summary; BKD, Burden of Kidney Disease; SPKD, Symptoms and Problems of Kidney Disease; EKD, Effects of Kidney Disease.

\section{RESULTS}

\section{Socioeconomic Characteristics of the Patients After Kidney Transplantation}

Out of the $153 \mathrm{KT}$ patients interviewed, 114 (74.51\%) were men. The age of patients ranged from 19 to 70 years, with an average age of $39.48 \pm 10.76$ years. They were predominantly living in urban areas (62.75\%) and were married (69.93\%). Out of these, $45.75 \%$ of patients were officers, $29.41 \%$ were freelancers, $11.11 \%$ were farmers, $7.19 \%$ were students, and $6.54 \%$ were retired. Most patients had the education of upper secondary or higher (93.46\%) (Table 1).

Of the post-KT patients, $60.78 \%$ were followed up for 1 year or more and $39.22 \%$ were followed up under 1 year. The mean time on dialysis was $38.45 \pm 88.33$ months. The most common comorbidities were hypertension (23.78\%) and diabetes (7.19\%). After KT, most patients reported facing an economic burden and $50.30 \%$ of them were severely affected (Table $\mathbf{1}$ ).

\section{Health-Related Quality of Life Measured by Use of the SF-36 and KDQOL-36}

The distribution of domain scores by the SF-36 and KDQOL36 scales was shown in Figure 1. We also examined the changes in the HRQOL scores in the domains at below 6 months, 6 
TABLE 2 | Component scores of the Short Form 36 (SF-36) and Kidney Disease Quality of Life 36 (KDQOL-36) by post-kidney transplant stages and Cronbach's alpha values of the domains.

\begin{tabular}{|c|c|c|c|c|c|c|}
\hline & $\begin{array}{l}\text { Below } 6 \text { months } \\
\qquad(n=39)\end{array}$ & $\begin{array}{l}6 \text { months to } 1 \\
\text { year }(n=21)\end{array}$ & $\begin{array}{c}1 \text { year to } 3 \text { years } \\
(n=64)\end{array}$ & $\begin{array}{c}\text { Above } 3 \text { years } \\
\qquad(n=29)\end{array}$ & Overall $(n=153)$ & Cronbach's $\alpha$ \\
\hline \multicolumn{7}{|l|}{ SF-36 } \\
\hline PF & $61.15 \pm 22.81$ & $67.86 \pm 17.79$ & $66.80 \pm 18.86$ & $60.34 \pm 22.56$ & $64.28 \pm 20.56$ & 0.84 \\
\hline $\mathrm{RP}$ & $41.67 \pm 41.49$ & $61.90 \pm 45.15$ & $62.50 \pm 40.58$ & $56.90 \pm 44.27$ & $56.04 \pm 42.63$ & 0.88 \\
\hline $\mathrm{BP}$ & $71.41 \pm 19.46$ & $81.43 \pm 16.98$ & $80.62 \pm 20.04$ & $84.91 \pm 16.72$ & $79.20 \pm 19.34$ & 0.76 \\
\hline $\mathrm{GH}$ & $81.67 \pm 11.49$ & $83.09 \pm 11.88$ & $78.28 \pm 17.51$ & $79.65 \pm 14.26$ & $80.06 \pm 14.81$ & 0.60 \\
\hline VT & $75.21 \pm 11.71$ & $79.05 \pm 13.19$ & $75.60 \pm 14.46$ & $71.90 \pm 15.83$ & $75.27 \pm 13.94$ & 0.44 \\
\hline SF & $59.61 \pm 17.78$ & $64.28 \pm 20.65$ & $65.23 \pm 16.59$ & $66.38 \pm 17.39$ & $63.89 \pm 17.65$ & 0.45 \\
\hline RE & $49.57 \pm 42.49$ & $57.14 \pm 48.49$ & $55.21 \pm 43.33$ & $57.47 \pm 42.63$ & $54.47 \pm 43.38$ & 0.84 \\
\hline $\mathrm{MH}$ & $83.10 \pm 11.98$ & $82.09 \pm 16.23$ & $80.11 \pm 14.86$ & $80.55 \pm 14.76$ & $81.23 \pm 14.28$ & 0.65 \\
\hline Total & $65.93 \pm 15.13$ & $72.43 \pm 15.41$ & $70.59 \pm 15.08$ & $67.82 \pm 17.08$ & $69.13 \pm 15.55$ & 0.90 \\
\hline \multicolumn{7}{|c|}{ KDQOL-36 } \\
\hline PCS & $65.64 \pm 24.23$ & $79.28 \pm 16.30$ & $69.37 \pm 24.90$ & $74.83 \pm 22.58$ & $70.82 \pm 23.52$ & 0.66 \\
\hline MCS & $70.88 \pm 17.16$ & $72.35 \pm 21.16$ & $71.13 \pm 20.90$ & $71.72 \pm 9.46$ & $71.34 \pm 19.59$ & 0.72 \\
\hline BKD & $50.64 \pm 26.08$ & $51.78 \pm 29.19$ & $54.59 \pm 25.47$ & $55.82 \pm 22.90$ & $53.43 \pm 25.53$ & 0.83 \\
\hline SPKD & $84.91 \pm 16.43$ & $90.80 \pm 15.25$ & $89.13 \pm 14.40$ & $82.68 \pm 18.56$ & $87.06 \pm 16.00$ & 0.92 \\
\hline EKD & $48.02 \pm 28.85$ & $44.79 \pm 27.64$ & $49.61 \pm 32.34$ & $43.21 \pm 27.04$ & $47.32 \pm 29.72$ & 0.93 \\
\hline Total & $67.00 \pm 13.26$ & $70.49 \pm 14.20$ & $69.73 \pm 14.06$ & $67.27 \pm 13.67$ & $68.67 \pm 13.75$ & 0.88 \\
\hline
\end{tabular}

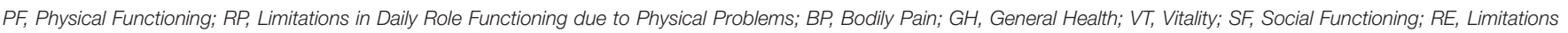

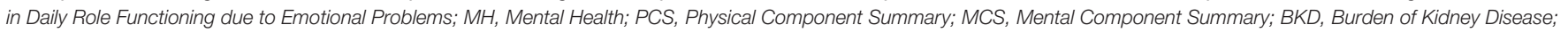
SPKD, Symptoms and Problems of Kidney Disease; EKD, Effects of Kidney Disease. Period after kidney transplant having highest component score was given in bold.

months -1 year, $1-3$ years, and above 3 years after transplantation (Table 2). According to the SF-36, the overall mean score of HRQOL was $69.13 \pm 15.55$ and the two dimensions were the highest scores of MH $(81.23 \pm 14.28)$ and GH $(80.06 \pm 14.81)$. Two dimensions of RP and RE were observed with the lowest mean scores of $56.04 \pm 42.63$ and $54.47 \pm 43.38$, respectively. In particular, the score increases gradually in $\mathrm{BP}, \mathrm{SF}$, and $\mathrm{RE}$ over time and their highest scores were observed after 3-year transplantation, at $84.91 \pm 16.72,66.38 \pm 17.39$, and 57.47 \pm 42.63 , respectively. The remaining dimensions of HRQOL commonly had the highest scores in the short-term duration within 6-12 months (Table 2).

When measuring with the KDQOL-36, the overall mean score was $68.67 \pm 13.75$ and was the highest in the SPKD $(87.06 \pm$ 16.00). Two dimensions of the BKD and EKD had the lowest mean scores of all with $53.43 \pm 25.53$ and $47.32 \pm 29.72$, respectively. HRQOL domain scores measured by KDQOL-36 score were mainly high at 6 months to less than 1 year after KT. The BKD score has increased over time, reflective of a lower perceived BKD (Table 2).

\section{Validity and Reliability of the SF-36 and KDQOL-36}

As indicated in Table 1, both the tools showed good reliability when used in our patients after KT. The reliability of the SF36 was high with Cronbach's coefficients $\alpha=0.90$. Internal consistency in all domains was adequate (Cronbach's $\alpha \geq 0.60$ ), except for vitality (VT) (Cronbach's $\alpha=0.44$ ) and social activities (Cronbach $\alpha=0.45$ ). The reliability of the KDQOL-36 scale was overall high with Cronbach's coefficients $\alpha=0.88$, while Cronbach's $\alpha$ in domains ranged from 0.66 to 0.93 .

Correlations between the domains of SF-36 and KDQOL-36 are pointed out in Figures 2, 3. In general, there were positive relationships between the dimensions assessed by the KDQOL36 and SF-36 (correlation coefficient: 0.03-0.69). Similarly, the domains of the SF-36 also had positive correlations with KDQOL-36 (correlation coefficient: 0.18-0.51).

The correlation coefficient between overall HRQOL scores of SF-36 and KDQOL-36 was 0.62 , indicating a strong correlation between SF-36 and KDQOL-36. The comprehensive distribution of the correlation between the SF-36 and the KDQOL-36 is illustrated in Figure 4.

\section{DISCUSSION}

In addition to helping the patients in achieving optimal health compared to before the onset of disease, the patients after transplantation also need to ensure the balance between the function of organ transplant and the psychological integrity and physical integrity. This study highlights the importance of assessing the HRQOL in the KT patient after treatment, thereby determining the effectiveness of the instruments for the assessment of HRQOL in KT patients.

The study shows that the HRQOL of patients after KT fluctuated above average, both physical and mental health. The scores in each dimension changed according to the follow-up timelines within 3 years after transplantation but did not show a clear trend of change. The average scores generally obtained 


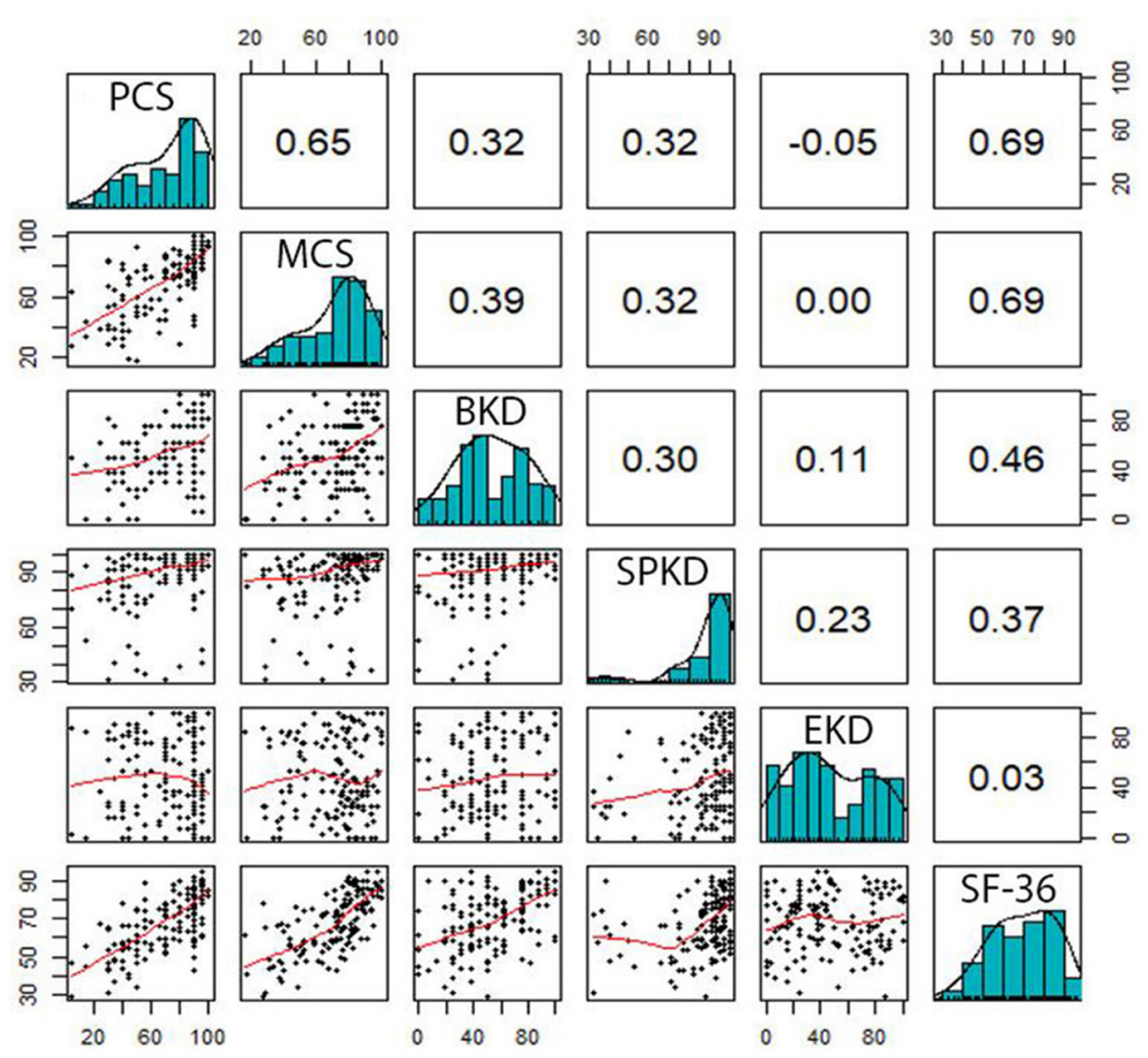

FIGURE 2 | Correlation between the domains measured by the SF-36 and KDQOL-36. PCS, Physical Component Summary; MCS, Mental Component Summary; BKD, Burden of Kidney Disease; SPKD, Symptoms and Problems of Kidney Disease; EKD, Effects of Kidney Disease.

from the SF-36 and KDQOL-36 were quite similar (69.13 \pm 15.55 compared to $68.67 \pm 13.75$ ). This is consistent with the recent report of Cordeiro in Brazil on 222 individuals after renal transplantation showing the average score by SF-36 and KDQOL of $64.7 \pm 24.3$ and $78.5 \pm 16.8$, respectively (13). An early report by Humar in 2,746 post-KT patients in the United States recorded higher scores with all domains above 60 points, highest in two health aspects, namely PF and RP (>90 points) (14). Compared with the individuals with ESKD who receive dialysis treatment in several previous studies in Vietnam, the overall HRQOL scores were 40.78 points in 112 patients at 103 Military Hospital and 48.89 points in 360 patients at People's Hospital 115 (9). Compared to the scores of physical and psychosocial domains of HRQOL in the previous reports $(15,16)$, there was a significant increase after transplant among most domains in our study. Though HRQOL of KT patients in Vietnam is not as high as in developed countries, the current result has improved significantly as compared to the patients with end-stage renal disease on hemodialysis. Among various alternative treatments for chronic kidney failure, most studies agree that KT significantly improves the HRQOL for patients (17-19).

When being assessed physically and mentally, patients reported that their HRQOL was not significantly affected, with average scores hovering over 50 points in all relevant domains.
Meanwhile, when examining the effects of kidney disease using the KDQOL-36 specific tool, we obtained a relatively low score for the $\mathrm{EKD}$ ( $<50$ points during follow-up), while BKD was reported to be $53.43 \pm 25.53$, with a high domain score of SPKD $(87.06 \pm 16.00)$. The findings presented in this study support the conclusion that the present treatment was capable of improving completely a number of symptoms and problems of kidney disease; however, the patient still finds it difficult to adapt their lives to new conditions partially due to monthly examination and lifelong antirejection medications. The previous studies reported that the specific domain scores of kidney disease were quite high, 78.5 in BKD, 81.7 in SPKD, and 83.8 in EKD (20), which were better scores as compared to this study. The differences between the studies may be mainly explained by a resource-scarce condition in clinical practice, economic hardship on the patient and their family, and aspects related to trust and belief. The current evidence supported the point that the conditions to support and care for post-KT Vietnamese patients are still not optimal as compared to the developed countries.

In the KDQOL-36 scale, the average total score of 12 questions on PCS and MCS, questions were shortened from the SF36 , quite similar to the SF-36 score. The present analysis showed that two questionnaires of the SF-36 and KDQOL-36 


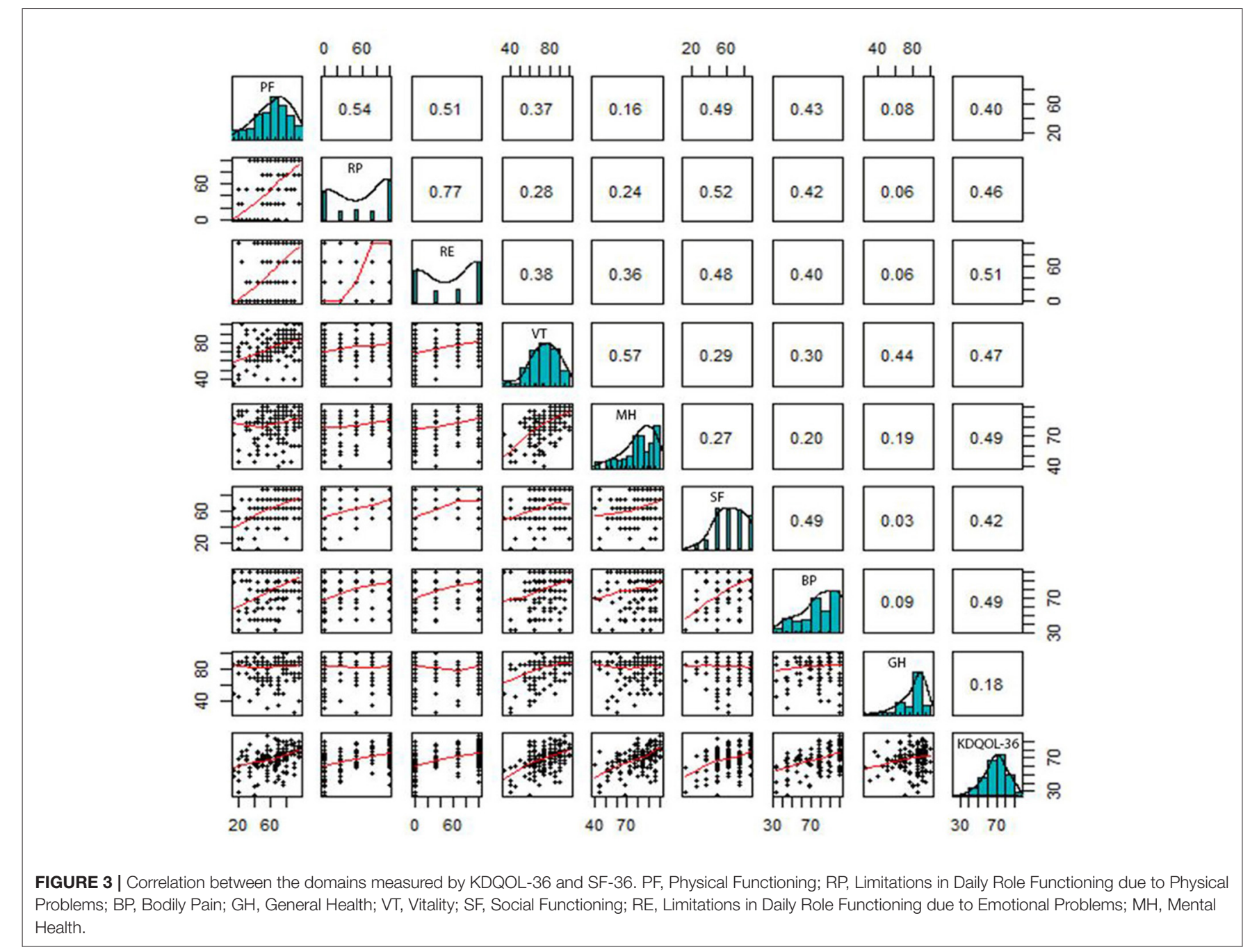

are highly reliable (Cronbach's $\alpha$ : 0.90 and 0.88 , respectively) with a moderate positive relationship (correlation coefficient $=0.62$ ), which showed that both scales giving the HRQOL scores were fairly comparable. The SF-36 questionnaire has been proven in many different healthcare settings, with various patient populations, so it is very effective for general HRQOL assessment and HRQOL comparison among multiple groups of subjects. However, it can be seen that the contents of the SF-36 scale do not help in making judgments about specific aspects in a particular population such as chronic kidney disease in this cohort, while 24 concentrated questions of the kidney disease known as the KDQOL-36 scale enable us to give a more accurate view. Disease-specific questionnaires have the advantage of focusing more on relevant aspects with high sensitivity in the changes in the clinical condition of that disease over time, thereby it is more practical for both physicians and patients (17). To our knowledge, there have been no identified questionnaires yet that can optimally measure all aspects of concern, while the questionnaires measuring HRQOL also can give mixed results once using on the same group of subjects (4). To our knowledge, it is not yet clear which instruments can measure all aspects of concern, while the instruments of HRQOL when used on the same patient group still commonly give different results (4). Current findings suggest that to make an HRQOL assessment of an individual after KT, it is possible to use either the SF-36 or the KDQOL36 instruments to give the same results, but the results from this study also implied the need of depending on the specific aspect of HRQOL that the researcher care to choose the right instrument.

The results of this study should be interpreted within the context of some limitations. First our study is not powered to demonstrate the significance of two instruments in kidney disease-specific HRQOL domains. Second although the study did not evaluate the changes in HRQOL over time after KT vs. pre-KT, these were also valuable reference results to initially evaluate and determine the aspects of HRQOL in Vietnamese patients after KT. Future longitudinal studies are necessary to more comprehensively evaluate the process of HRQOL changes and their associated factors in the patients before and after KT, 


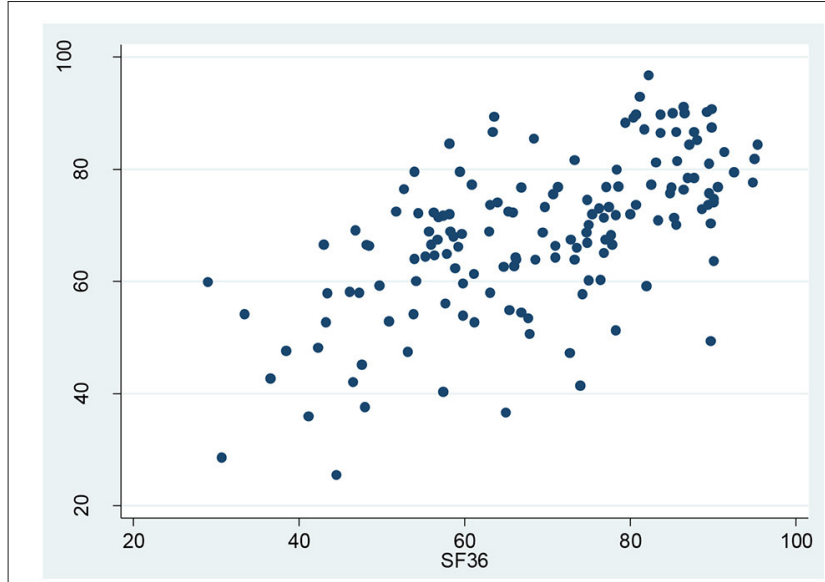

FIGURE 4 | Correlation between the overall health-related quality of life (HRQOL) scores of the KDQOL-36 and SF-36.

thereby improving the quality of treatment and increasing the ability in the detection of specific symptoms and subtle changes in the quality of life. Finally, the translated Vietnamese version of the KDQOL-36 scale has not been evaluated by RAND, and its psychometric properties have not been confirmed in a representative sample of the population; therefore, a proper translation of the KDQOL-36 scale may not guarantee that the scale is reliable and valid in the population.

\section{CONCLUSIONS}

We found that there were slight fluctuations in the HRQOL score in domains within 3-year follow-up after KT. The mean

\section{REFERENCES}

1. Coons SJ, Rao S, Keininger DL, Hays RD. A comparative review of generic quality-of-life instruments. Pharmacoeconomics. (2000) 17:13-35. doi: 10.2165/00019053-200017010-00002

2. Webster AC, Nagler EV, Morton RL, Masson P. Chronic kidney disease. Lancet. (2017) 389:1238-52. doi: 10.1016/S0140-6736(16)3 2064-5

3. Shetty AA, Wertheim JA, Butt Z. Health-related quality of life outcomes after kidney transplantation, in Kidney transplantation, bioengineering and regeneration. Elsevier (2017). p. 699-708. doi: 10.1016/B978-0-12-801734-0.0 0050-3

4. Fiebiger W, Mitterbauer C, Oberbauer R. Health-related quality of life outcomes after kidney transplantation. Health Qual Life Outcomes. (2004) 2:1-6. doi: 10.1186/1477-7525-2-2

5. Maglakelidze N, Pantsulaia T, Tchokhonelidze I, Managadze L, Chkhotua A. Assessment of health-related quality of life in renal transplant recipients and dialysis patients. In Transplantation Proceedings. Elsevier (2011). p. 376-9. doi: 10.1016/j.transproceed.2010. 12.015

6. Tonelli M, Wiebe N, Knoll G, Bello A, Browne S, Jadhav D, et al. Systematic review: kidney transplantation compared with dialysis in clinically relevant outcomes. Am J Trans. (2011) 11:2093-109. doi: 10.1111/j.1600-6143.2011.03686.x
SF-36 score was consistent with the mean KDQOL-36 score. High reliability and strong correlation were documented between the two instruments of SF-36 and KDQOL-36. In the developing condition in the clinical practice of Vietnam, both the SF-36 and KDQOL-36 can be considered as suitable instruments for assessing the HRQOL in patients after KT but initially need to be selected based on specific aspects that the physicians want to learn.

\section{DATA AVAILABILITY STATEMENT}

The raw data supporting the conclusions of this article will be made available by the authors, without undue reservation.

\section{ETHICS STATEMENT}

The studies involving human participants were reviewed and approved by Ethics Board of the Hanoi Medical University. The patients/participants provided their written informed consent to participate in this study.

\section{AUTHOR CONTRIBUTIONS}

$\mathrm{LV}, \mathrm{NN}$, and TT have all made substantial contributions to conception and design of the study. TP and TT were responsible for the acquisition of data. LV, KV, and H-LV were responsible for analysis and interpretation of data. All authors were involved in drafting the manuscript or revising it critically for important intellectual content. All authors have given final approval of the version to be submitted.
7. Kostro JZ, Hellmann A, Kobiela J, Skóra I, LichodziejewskaNiemierko M, Debska-Slizień A, et al. Quality of life after kidney transplantation: a prospective study. In Transplantation Proceedings. Elsevier (2016). p. 50-4. doi: 10.1016/j.transproceed.2015. 10.058

8. Chan-On C, Sarwal MM. A comprehensive analysis of the current status and unmet needs in kidney transplantation in Southeast Asia. Front Med. (2017) 4:84. doi: 10.3389/fmed.2017.00084

9. Afendy A, Kallman JB, Stepanova M, Younoszai Z, Aquino RD, Bianchi $G$, et al. Predictors of health-related quality of life in patients with chronic liver disease. Aliment Pharmacol Ther. (2009) 30:469-76. doi: 10.1111/j.1365-2036.2009.04061.x

10. Kefale B, Alebachew M, Tadesse Y, Engidawork E. Quality of life and its predictors among patients with chronic kidney disease: A hospital-based cross sectional study. PLoS ONE. (2019) 14:e0212184. doi: 10.1371 /journal.pone. 0212184

11. RAND Health Care. Kidney Disease Quality of Life Instrument (KDQOL) Available online at: https://www.rand.org/health-care/surveys_tools/kdqol. html

12. Bland JM, Altman DG. Statistics notes: Cronbach's alpha. BMJ. (1997) 314:572. doi: 10.1136/bmj.314.7080.572

13. Cordeiro ED, Costa TC, Teixeira MF, Toledo ND, Almeida GS. Quality of life of individuals receiving kidney transplantation in Amazonas state. Rev Latino-Am Enfermagem. (2020) 28:e3291. doi: 10.1590/1518-8345.3775.3291 
14. Humar A, Denny R, Matas AJ, Najarian JS. Graft and quality of life outcomes in older recipients of a kidney transplant. Exper Clin Trans. (2003) 1:69-72. http://www.ectrx.org/forms/ectrxcontentshow.php?year=2003\&volume $=1 \&$ issue $=2 \&$ supplement $=0 \&$ makale_no $=0 \&$ spage_number $=69 \&$ content_type $=$ FULL\%20TEXT

15. Junchotikul P, Charoenthanakit C, Saiyud A, Parapiboon W, Ingsathit A, Jirasiritham S, et al. Assessment of the changes in health-related quality of life after kidney transplantation in a cohort of 232 Thai patients. In Transplantation Proceedings. Elsevier (2015). p. 1732-5. doi: 10.1016/j.transproceed.2015.02.018

16. Lim HJ, Koo TY, Lee J, Huh KH, Park JB, Cho J, et al. Health-related quality of life of kidney transplantation patients: results from the korean cohort study for outcome in patients with kidney transplantation (KNOWKT) study. In Transplantation Proceedings. Elsevier (2016). p. 844-7. doi: 10.1016/j.transproceed.2015.12.101

17. Butt Z, Yount SE, Caicedo JC, Abecassis MM, Cella D. Quality of life assessment in renal transplant: review and future directions. Clin Transplant. (2008) 22:292-303. doi: 10.1111/j.1399-0012.2007.0 0784.x

18. Tomasz W, Piotr S. A trial of objective comparison of quality of life between chronic renal failure patients treated with hemodialysis and renal transplantation. Ann Trans. (2003) 8:47. https://www. annalsoftransplantation.com/abstract/index/idArt/142803

19. Fujisawa M, Ichikawa $Y$, Yoshiya K, Isotani S, Higuchi A, Nagano S, et al. Assessment of health-related quality of life in renal transplant and hemodialysis patients using the SF-36 health survey. Urology. (2000) 56:201-6. doi: 10.1016/S0090-4295(00)00623-3

20. Aiyegbusi OL, Kyte D, Cockwell P, Marshall T, Gheorghe A, Keeley T, et al. Measurement properties of patient-reported outcome measures (PROMs) used in adult patients with chronic kidney disease: a systematic review. PLoS ONE. (2017) 12:e0179733. doi: 10.1371/journal.pone.0179733

Conflict of Interest: The authors declare that the research was conducted in the absence of any commercial or financial relationships that could be construed as a potential conflict of interest.

Publisher's Note: All claims expressed in this article are solely those of the authors and do not necessarily represent those of their affiliated organizations, or those of the publisher, the editors and the reviewers. Any product that may be evaluated in this article, or claim that may be made by its manufacturer, is not guaranteed or endorsed by the publisher.

Copyright (c) 2021 Vu, Nghia, Tuan, Phuong, Vo, Viet and Giang. This is an openaccess article distributed under the terms of the Creative Commons Attribution License (CC BY). The use, distribution or reproduction in other forums is permitted, provided the original author(s) and the copyright owner(s) are credited and that the original publication in this journal is cited, in accordance with accepted academic practice. No use, distribution or reproduction is permitted which does not comply with these terms. 